Vasile Ene*广, Ovidius University Constanța, Romania Current address ${ }^{\ddagger}$ : Quellenstrasse 18, 63571 Gelnhausen, Germany

e-mail: gabrielaene@hotmail.com

\title{
THOMSON'S VARIATIONAL MEASURE AND SOME CLASSICAL THEOREMS
}

\begin{abstract}
Using the conditions increasing* and decreasing*, and Thomson's variational measure, we give an easy proof of the Denjoy-Lusin-Saks Theorem [12, p. 230]. In Theorem 5.1 we extend (the function is not assumed to be continuous) Thomson's Theorems 44.1 and 44.2 of [13], that are closely related to the Denjoy-Lusin-Saks Theorem. From this extension we obtain another classical result: the Denjoy-Young-Saks Theorem [5]. As consequences of the Denjoy-Lusin-Saks Theorem we obtain two well-known results due to de la Vallée Poussin [12, p. 125, 127]. Then wee extend these results (the set $E$ used there is not only Borel, but also Lebesgue measurable) and give in Theorem 8.1 a de la Vallée Poussin type theorem for $V B^{*} G$ functions, that is in fact an extension of a result of Thomson [13, Theorem 46.3]. Finally, we give characterizations for Lebesgue measurable functions that are $V B^{*} G \cap$ $(N)$, and for measurable functions that are $V B^{*} G \cap N^{+\infty}$ on a Lebesgue measurable set.
\end{abstract}

\section{Introduction}

Using the conditions increasing* and decreasing*, and Thomson's variational measure, we give (see Corollary 5.1, (i), (iii)) an easy proof of the following theorem of Saks:

Key Words: Thomson's variational measure, the condition increasing* $V B^{*} G$, Lusin's condition $(N), F$-null sets

Mathematical Reviews subject classification: 26A45, 26A46, 26A24

Received by the editors January 4, 1999

* The author died on November 11.

†The author's wife wishes to thank the referee for the special attention he has taken in correcting all the details. It shows a nice measure of respect to the author's memory. The new proofs of the Lemmas 3.4, 5.2, 5.4, 5.5, 10.1, 10.3 and Theorem 8.1, and many useful comments have led to the improvement of this paper.

$¥$ At this address you can contact the author's wife Gabriela Ene. The author's web site is: www.vasile-ene.subdomain.de 
Theorem A. Let $F:[a, b] \rightarrow \mathbb{R}$ and $E \subset[a, b]$. If $F$ is $V B^{*} G$ on $E$, then $F$ is derivable a.e. on this set; and further if $N=\left\{x \in E: F^{\prime}(x)\right.$ does not exist (finite or infinite) $\}$, then $m(F(N))=\Lambda(B(F ; N))=0$.

Since for continuous functions, this result has been proved independently by Denjoy and Lusin [12, p. 230], we call it the Denjoy-Lusin-Saks Theorem. In Theorem 5.1 (see also Corollary 5.1 and Remark 5.2) we extend (the function is not assumed to be continuous) Thomson's Theorems 44.1 and 44.2 of [13], that are closely related to Theorem A. From Theorem 5.1 we obtain another classical result: the Denjoy-Young-Saks Theorem [5].

Using Theorem A we obtain the following results of de la Vallée Poussin.

Theorem B. ([12, p. 125]) For a function $F:[a, b] \rightarrow \mathbb{R}$ of bounded variation we have $\left|F^{*}(N)\right|=V_{F}^{*}(N)=m^{*}(N)=0$ and $\Lambda(B(F ; N))=0$, where $V_{F}$ is the total variation of $F$ and $N=\left\{x \in[a, b]: F\right.$ is continuous at $x, F^{\prime}(x)$ does not exist (finite or infinite) $\}$.

Note that in the book of Saks [12], the proof of the Denjoy-Lusin-Saks Theorem is based on Theorem B.

Theorem C. $\left(\left[12\right.\right.$, p. 127]) If $F:[a, b] \rightarrow \mathbb{R}, F \in V B$. Let $E_{+\infty}=\{x \in$ $\left.[a, b]: F^{\prime}(x)=+\infty\right\}, E_{-\infty}=\left\{x \in[a, b]: F^{\prime}(x)=-\infty\right\}$, and let $V_{F}$ be the total variation of $F$.

(i) If $X$ is a Borel measurable subset of $[a, b]$ and if $F$ is continuous at each point of $X$, then

$$
F^{*}(X)=F^{*}\left(X \cap E_{+\infty}\right)+F^{*}\left(X \cap E_{-\infty}\right)+\int_{X} F^{\prime}(x) d t,
$$

and

$$
V_{F}^{*}(X)=F^{*}\left(X \cap E_{+\infty}\right)+\left|F^{*}\left(X \cap E_{-\infty}\right)\right|+\int_{X}\left|F^{\prime}(x)\right| d x .
$$

(ii) Let $E=\left\{x \in[a, b]: F\right.$ is continuous at $x, F^{\prime}$ and $V_{F}^{\prime}$ exist (finite or infinite), $\left.V_{F}^{\prime}(x)=\left|F^{\prime}(x)\right|\right\}$. Then $V_{F}^{*}([a, b] \backslash E)=m^{*}([a, b] \backslash E)=0$.

In fact Theorem 7.2, (vii), (viii), (ix) is an extension of Theorem $\mathrm{C}$ (because in (vii) and (viii) the set $E$ is not only Borel but also Lebesgue measurable). Note also that in order to prove Theorem C, Saks uses the Lebesgue Decomposition Theorem [12, p. 119], whereas our proof does not use this decomposition; it is instead essentially based on Theorem 8.2 of [4] (see Lemma 3.2). 
In Theorem 8.1 we give a de la Vallée Poussin type theorem for $V B^{*} G$ function, that is in fact an extension of a result of Thomson [13, Theorem 46.3].

Finally, as consequences of the previous results, we give characterizations: for Lebesgue measurable functions that are $V B^{*} G \cap(N)$, and for measurable functions that are $V B^{*} G \cap N^{+\infty}$ on a Lebesgue measurable set.

\section{Preliminaries}

Let $m^{*}(X)$ denote the outer measure of the set $X$ and $m(E)$ the Lebesgue measure of $E$, whenever $E \subseteq \mathbb{R}$ is Lebesgue measurable. For the definitions of $V B$, $V B^{*}, V B^{*} G$ and Lusin's condition $(N)$, see [12]. We denote by $\mathcal{O}(F ;[a, b])$ the oscillation of the function $F$ on the closed interval $[a, b]$. Let int $(E)$ denote the interior of the set $E$.

Definition 2.1. Let $F:[a, b] \rightarrow \mathbb{R}, E \subseteq[a, b]$. We denote by $V^{*}(F ; E)=$ $\left\{\sum_{k=1}^{n} \mathcal{O}\left(F ;\left[a_{k}, b_{k}\right]\right):\left\{\left[a_{k}, b_{k}\right]\right\}_{k=1}^{n}\right.$ is a finite set of nonoverlapping closed intervals with $\left.a_{k}, b_{k} \in E\right\}$.

Definition 2.2. [12, p 64.] Let $F: \mathbb{R} \rightarrow \mathbb{R}$ be an increasing function. For each set $E \subset \mathbb{R}$, let

$$
F^{*}(E)=\inf \left\{\sum_{i=1}^{\infty}\left(F\left(b_{i}\right)-F\left(a_{i}\right)\right): E \subset \cup_{i=1}^{\infty}\left(a_{i}, b_{i}\right)\right\} .
$$

Lemma 2.1. [12] Let $F^{*}$ be defined as in Definition 2.2, and let $E \subset \mathbb{R}$.

(i) $F^{*}$ is a metric outer measure (or with the notations of [12, p. 64], $F^{*}$ is an outer measure in the sense of Caratheodory).

(ii) All Borel measurable sets of $\mathbb{R}$ are $F^{*}$-measurable; i.e.,

$$
F^{*}(X)=F^{*}(X \cap B)+F^{*}(X \backslash B)
$$

whenever $B$ is a Borel set and $X \subset \mathbb{R}$.

(iii) For every $\epsilon>0$, there is an open set $G$ that contains $E$ such that $F^{*}(G) \leq$ $F^{*}(E)+\epsilon$.

(iv) $F^{*}(E)=\inf \left\{F^{*}(G): G\right.$ is an open set that contains $\left.E\right\}$.

(v) If $F$ is continuous at each point of $E$, then $F^{*}(E)=m^{*}(F(E))$.

(vi) $F^{*}(A)=F(b-)-F(a+)$ for $A=(a, b)$, and $F^{*}(A)=F(b+)-F(a-)$ for $A=[a, b]$. 
Proof. (i) See [12, p. 64].

(ii) See Theorem 7.4 of $[12$, p. 52$]$.

(iii) See Theorem 6.5 , (i) of $[12$, p. 68].

(iv) See (iii).

(v) See [12, p. 100].

(vi) This is evident.

Definition 2.3. Let $F:[a, b] \rightarrow \mathbb{R}$. For $x, y \in[a, b], x<y$, let

$$
\begin{gathered}
\Delta F^{+}([x, y])=\max \{F(y)-F(x), 0\} \text { and } \\
\Delta F^{-}([x, y])=\max \{F(x)-F(y), 0\} .
\end{gathered}
$$

Clearly

$$
|F(y)-F(x)|=\Delta F^{+}([x, y])+\Delta F^{-}([x, y]) .
$$

Definition 2.4. [8, p. 51-52].

Let $F:[a, b] \rightarrow \mathbb{R}$. For each $x \in(a, b]$ let

$$
\begin{aligned}
& V(F ;[a, x])=\sup \left\{\sum_{i=1}^{n}\left|F\left(x_{i}\right)-F\left(x_{i-1}\right)\right|: a=x_{0}<x_{1}<\ldots<x_{n}=x\right\} ; \\
& \bar{V}(F ;[a, x])=\sup \left\{\sum_{i=1}^{n} \Delta F^{+}\left(\left[x_{i-1}, x_{i}\right]\right): a=x_{0}<x_{1}<\ldots<x_{n}=x\right\} ; \\
& \underline{V}(F ;[a, x])=\sup \left\{\sum_{i=1}^{n} \Delta F^{-}\left(\left[x_{i-1}, x_{i}\right]\right): a=x_{0}<x_{1}<\ldots<x_{n}=x\right\} .
\end{aligned}
$$

Consider $F: \mathbb{R} \rightarrow \mathbb{R}$ where $F(x)=F(a)$ for $x<a$ and $F(x)=F(b)$ for $x>b$. Let's put

$$
\begin{gathered}
V_{F}: \mathbb{R} \rightarrow \mathbb{R}, \quad V_{F}(x)= \begin{cases}0 & \text { if } x \in(-\infty, a] \\
V(F ;[a, x]) & \text { if } x \in(a, b] \\
V(F ;[a, b]) & \text { if } x \in(b,+\infty)\end{cases} \\
\bar{V}_{F}: \mathbb{R} \rightarrow \mathbb{R}, \quad \bar{V}_{F}(x)= \begin{cases}0 & \text { if } x \in(-\infty, a] \\
\bar{V}(F ;[a, x]) & \text { if } x \in(a, b] \\
\bar{V}(F ;[a, b]) & \text { if } x \in(b,+\infty)\end{cases} \\
\underline{V}_{F}: \mathbb{R} \rightarrow \mathbb{R}, \quad \underline{V}_{F}(x)= \begin{cases}0 & \text { if } x \in(-\infty, a] \\
\underline{V}(F ;[a, x]) & \text { if } x \in(a, b] \\
\underline{V}(F ;[a, b]) & \text { if } x \in(b,+\infty)\end{cases}
\end{gathered}
$$

Clearly $\underline{V}_{F}=\bar{V}_{-F}$. 
Thomson's Variational Measure and Some Classical Theorems 525

Remark 2.1. Note that

$$
\begin{aligned}
& \bar{V}(F ;[a, x])=\bar{W}(F ;[a, x])=W_{1}([a, x]), \\
& \underline{V}(F ;[a, x])=-\underline{W}(F ;[a, x])=-W_{2}([a, x]) \text { and } \\
& V(F ;[a, x])=W(F ;[a, x])=W([a, x])
\end{aligned}
$$

where the "W" variants are those defined in $[12, \mathrm{p} 61]$.

Theorem 2.1. $[8$, p. 52] Let $F:[a, b] \rightarrow \mathbb{R}, F \in V B$. Then for $x \in[a, b]$ we have

$$
\begin{aligned}
F(x)-F(a) & =\bar{V}(F ;[a, x])-\underline{V}(F ;[a, x]) \text { and } \\
V(F ;[a, x]) & =\bar{V}(F ;[a, x])+\underline{V}(F ;[a, x]) .
\end{aligned}
$$

Thus, if one of the three numbers $V(F ;[a, x]), \bar{V}(F ;[a, x]), \underline{V}(F ;[a, x])$ is finite, then the other two are also finite.

Definition 2.5. [12, p. 64]. Let $F: \mathbb{R} \rightarrow \mathbb{R}, F \in V B$ on $[a, b], F$ is constant on $(-\infty, a]$ and on $[b,+\infty)$. For each $E \subset \mathbb{R}$, let

$$
F^{*}(E)=\bar{V}_{F}^{*}(E)-\underline{V}_{F}^{*}(E) .
$$

Lemma 2.2. Let $F_{1}, F_{2}: \mathbb{R} \rightarrow \mathbb{R}$ be increasing functions, and let $E \subset \mathbb{R}$. Then

$$
\left(F_{1}+F_{2}\right)^{*}(E)=F_{1}^{*}(E)+F_{2}^{*}(E) .
$$

In particular, we have $V_{F}^{*}(E)=\bar{V}_{F}^{*}(E)+\underline{V}_{F}^{*}(E)$.

Proof. If $A=(a, b)$, then by Lemma 2.1, (vi) we have

$$
\begin{gathered}
\left(F_{1}+F_{2}\right)^{*}(A)=\left(F_{1}+F_{2}\right)(b-)+\left(F_{1}+F_{2}\right)(a+)= \\
F_{1}(b-)-F_{1}(a+)+F_{2}(b-)-F_{2}(a+)=F_{1}^{*}(A)+F_{2}^{*}(A) .
\end{gathered}
$$

Now by Lemma 2.1 , (ii), if $B$ is an open set we have

$$
\left(F_{1}+F_{2}\right)^{*}(B)=F_{1}^{*}(B)+F_{2}^{*}(B) .
$$

Let $G_{1}$ and $G_{2}$ be open sets containing $E$, and let $G=G_{1} \cap G_{2}$. Then

$$
\left(F_{1}+F_{2}\right)^{*}(E) \leq\left(F_{1}+F_{2}\right)^{*}(G)=F_{1}^{*}(G)+F_{2}^{*}(G) \leq F_{1}^{*}\left(G_{1}\right)+F_{2}^{*}\left(G_{2}\right),
$$

and by Lemma 2.1, (iv), it follows that $\left(F_{1}+F_{2}\right)^{*}(E) \leq F_{1}^{*}(E)+F_{2}^{*}(E)$. Let $D$ be an open set that contains $E$. Then

$$
F_{1}^{*}(E)+F_{2}^{*}(E) \leq F_{1}^{*}(D)+F_{2}^{*}(D)=\left(F_{1}+F_{2}\right)^{*}(D) .
$$

Again by Lemma 2.1, (iv), we obtain that $F_{1}^{*}(E)+F_{2}^{*}(E) \leq\left(F_{1}+F_{2}\right)^{*}(E)$. 


\section{Thomson's Variational Measure}

Definition 3.1. Let $F: \mathbb{R} \rightarrow \mathbb{R}, E \subset \mathbb{R}, \delta: E \rightarrow(0,+\infty)$ and

$$
\beta_{\delta}^{*}(E)=\{(\langle x, y\rangle, x): x \in E, y \subset(x-\delta(x), x+\delta(x))\} .
$$

A set $\pi=\left\{\left(\left\langle x_{i}, y_{i}\right\rangle, x_{i}\right)\right\}_{i=1}^{n}$, with int $\left\langle x_{i}, y_{i}\right\rangle \cap \operatorname{int}\left\langle x_{j}, y_{j}\right\rangle=\emptyset$ for $i \neq j$, is said to be a partition. Let

$$
\begin{aligned}
V_{\delta}^{*}(F ; E)=\sup \left\{\sum_{i=1}^{n}\left|F\left(y_{i}\right)-F\left(x_{i}\right)\right|: \pi=\right. & \left\{\left(\left\langle x_{i}, y_{i}\right\rangle, x_{i}\right)\right\}_{i=1}^{n} \\
& \text { is a partition, } \left.\pi \subset \beta_{\delta}^{*}(E)\right\},
\end{aligned}
$$

and let $\mu_{F}^{*}(E)=\inf _{\delta} V_{\delta}^{*}(F ; E)$. Note that $\mu_{F}^{*}$ is in fact Thomson's variational measure $\mathcal{S}_{O^{-}} \mu_{F}$ defined in [13].

Lemma 3.1. Let $E \subset \mathbb{R}$. With the notations of Definition 3.1 we have:

(i) $\mu_{F}^{*}$ is a metric outer measure.

(ii) All Borel measurable sets of $\mathbb{R}$ are $\mu_{F}^{*}$-measurable; i.e.

$$
\mu_{F}^{*}(X)=\mu_{F}^{*}(X \cap B)+\mu_{F}^{*}(X \backslash B)
$$

whenever $B$ is a Borel set and $X \subset \mathbb{R}$.

(iii) If $F$ is increasing on $\mathbb{R}$ and $F$ is continuous at each point of $E$, then $\mu_{F}^{*}(E)=m^{*}(F(E))$.

(iv) For each $x \in E$ we have

$$
\mu_{F}^{*}(\{x\})=\limsup _{t \rightarrow 0+}|F(x+t)-F(x)|+\limsup _{t \rightarrow 0-}|F(x+t)-F(x)| .
$$

So, if $F$ is increasing in a neighborhood of $x$, then

$$
\mu_{F}^{*}(\{x\})=F(x+)-F(x-) .
$$

(v) If $F$ is $V B$ on $[a, b]$ and constant on each of the intervals $(-\infty, a]$ and $[b,+\infty)$, then $\mu_{F}^{*}(E)=\mu_{V_{F}}^{*}(E)$.

(vi) $m^{*}(F(E)) \leq \mu_{F}^{*}(E)$. 
Proof. (i) See [13, p. 40].

(ii) See Theorem 7.4 of $[12$, p. 52].

(iii) This follows easily.

(iv) See $[13$, p. 87].

(v) See $[13$, p. 92]

(vi) See [13, p. 101].

We denote by $C_{F}$ the set of continuity points of the function $F$.

Lemma 3.2. [4, Theorem 8.2]. Let $F:[a, b] \rightarrow \mathbb{R}$ and let $E$ be a Lebesgue measurable subset of $[a, b]$. If $F \in V B^{*} G \cap(N)$ on $E$, then

$$
\mu_{F}\left(E \cap C_{F}\right)=(\mathcal{L}) \int_{E}\left|F^{\prime}(t)\right| d t
$$

Lemma 3.3. [4, Corollary 6.1]. Let $F, G:[a, b] \rightarrow \mathbb{R}, E \subseteq[a, b]$. If $F, G \in$ $V B^{*}$ on $E$ and $F=G$ on $E$, then

$$
\mu_{F}^{*}\left(E \cap C_{F} \cap C_{G}\right)=\mu_{G}^{*}\left(E \cap C_{F} \cap C_{G}\right) .
$$

Lemma 3.4. Let $F:[a, b] \rightarrow \mathbb{R}$ and $E \subseteq[a, b]$. If $F$ is increasing on $[a, b]$, then $\mu_{F}^{*}\left(E \cap C_{F}\right)=m^{*}\left(F\left(E \cap C_{F}\right)\right)$.

Proof. This follows immediately by Lemma 3.1, (iii).

\section{The Conditions increasing*, decreasing* and VB*}

Definition 4.1. ([7], $[2$, p. 47]) Let $F:[a, b] \rightarrow \mathbb{R}, E \subset[a, b], c=\inf E$, $d=\sup E . \quad F$ is said to be increasing* (respectively decreasing*) on $E$ if $F(x) \leq F(y)$ (respectively $F(x) \geq F(y)$ ) whenever $c \leq x<y \leq d$ and $\{x, y\} \cap E \neq \emptyset$. $F$ is said to be increasing* $G$ (respectively decreasing* ${ }^{*} \mathrm{G}$ ) on $E$ if there is a sequence of sets $\left\{E_{n}\right\}$ such that $E=\cup_{n} E_{n}$ and $F$ is increasing* (respectively decreasing ${ }^{*}$ ) on each $E_{n}$. Note that the condition increasing* was introduced by Krzyzewski. See also the related condition "increasing around a set" of Thomson [13, p. 122].

Remark 4.1. Let $F:[a, b] \rightarrow \mathbb{R}, E \subset[a, b], c=\inf E, d=\sup E$. Note that if $F$ is increasing* on $E$, then $V^{*}(F ; E) \leq F(d)-F(c)$, so $F \in V B^{*}$ on $E$.

Lemma 4.1. Let $F: \mathbb{R} \rightarrow \mathbb{R}$, and let $E$ be a bounded set, $c=\inf E, d=\sup E$. The following assertions are equivalent.

(i) $F \in V B^{*}$ on $E$; 
(ii) $\sup \left\{\sum_{i=1}^{n}\left|F\left(d_{i}\right)-F\left(c_{i}\right)\right|:\left\{\left[c_{i}, d_{i}\right]\right\}_{i=1}^{n}\right.$ is a finite set of nonoverlapping closed intervals contained in $\left.[c, d],\left\{c_{i}, d_{i}\right\} \cap E \neq \emptyset\right\}<+\infty$;

(iii) $\sup \left\{\sum_{i=1}^{n} \Delta F^{+}\left(\left[c_{i}, d_{i}\right]\right):\left\{\left[c_{i}, d_{i}\right]\right\}_{i=1}^{n}\right.$ is a finite set of nonoverlapping closed intervals contained in $\left.[c, d],\left\{c_{i}, d_{i}\right\} \cap E \neq \emptyset\right\}<+\infty$;

(iv) $\sup \left\{\sum_{i=1}^{n} \Delta F^{-}\left(\left[c_{i}, d_{i}\right]\right):\left\{\left[c_{i}, d_{i}\right]\right\}_{i=1}^{n}\right.$ is a finite set of nonoverlapping closed intervals contained in $\left.[c, d],\left\{c_{i}, d_{i}\right\} \cap E \neq \emptyset\right\}<+\infty$;

(v) There exist $F_{1}, F_{2}:[c, d] \rightarrow \mathbb{R}$ increasing* on $E$ such that $F=F_{1}-F_{2}$.

Proof. (i) $\Rightarrow$ (ii) Let $\left\{\left[c_{i}, d_{i}\right]\right\}_{i=1}^{n}$ be a finite set of nonoverlapping closed subintervals of $[c, d]$, with $\left\{c_{i}, d_{i}\right\} \cap E \neq \emptyset$. Let $\mathcal{A}_{1}=\left\{i: c_{i} \in E\right\}$ and $\mathcal{A}_{2}=\left\{i: c_{i} \notin E\right\}$. Suppose that $\mathcal{A}_{1}=\left\{i_{1}, i_{2}, \ldots, i_{p}\right\}, p \leq n$ and $c_{i_{1}}<c_{i_{2}}<$ $\ldots<c_{i_{p}}$. Then

$$
\sum_{i \in \mathcal{A}_{1}}\left|F\left(d_{i}\right)-F\left(c_{i}\right)\right| \leq \sum_{k=1}^{p-1} \mathcal{O}\left(F ;\left[c_{i_{k}}, c_{i_{k+1}}\right]\right)+\mathcal{O}\left(F ;\left[c_{i_{p}}, d\right]\right) \leq V^{*}(F ; \bar{E}) .
$$

Similarly $\sum_{i \in \mathcal{A}_{2}}\left|F\left(d_{i}\right)-F\left(c_{i}\right)\right|<V^{*}(F ; \bar{E})$. Thus

$$
\sum_{i=1}^{n}\left|F\left(d_{i}\right)-F\left(c_{i}\right)\right| \leq 2 V^{*}(F ; \bar{E}) \neq+\infty
$$

(see [12, p. 229]), so we have (ii).

(ii) $\Rightarrow$ (iii) and (ii) $\Rightarrow$ (iv) are evident, because

$$
\left|F\left(d_{i}\right)-F\left(c_{i}\right)\right|=\Delta F^{+}\left(\left[c_{i}, d_{i}\right]\right)+\Delta F^{-}\left(\left[c_{i}, d_{i}\right]\right) .
$$

(iii) $\Rightarrow$ (v) Let $F_{1}:[c, d] \rightarrow \mathbb{R}, F_{1}(c)=0$, and for each $x \in(c, d]$, let

$F_{1}(x)=\sup \left\{\sum_{k=1}^{n} \Delta F^{+}\left(\left[a_{k}, b_{k}\right]\right):\left\{\left[a_{k}, b_{k}\right]\right\}_{k=1}^{n}\right.$ is a finite set of nonoverlapping closed intervals with $\left\{a_{k}, b_{k}\right\} \cap E \neq \emptyset$ and $\left.\left[a_{k}, b_{k}\right] \subset[c, x]\right\}$.

Let $F_{2}:[c, d] \rightarrow \mathbb{R}, F_{2}(x)=F_{1}(x)-F(x)$. Consider $x, y \in[c, d], x<y$ with $\{x, y\} \cap E \neq \emptyset$. Then

$$
F_{1}(y)-F_{1}(x) \geq \Delta F^{+}([x, y]) \geq F(y)-F(x),
$$

so $F_{1}(y)-F_{1}(x) \geq 0$ and $F_{2}(y)-F_{2}(x) \geq 0$. Therefore $F_{1}$ and $F_{2}$ are increasing* on $E$ and $F=F_{1}-F_{2}$ on $[c, d]$.

(iv) $\Rightarrow$ (v) The proof is similar to that of (iii) $\Rightarrow$ (v).

(v) $\Rightarrow$ (i) By Remark 4.1, $F_{1}$ and $F_{2}$ are $V B^{*}$ on $E$, so $F$ is $V B^{*}$ on $E$. 
Lemma 4.2. Let $F:[a, b] \rightarrow \mathbb{R}, E \subset[a, b], c=\inf E, d=\sup E$. Then $F$ is increasing ${ }^{*}$ on $E$ if and only if there exist $H_{1}, H_{2}:[c, d] \rightarrow \mathbb{R}$ increasing on $[c, d]$ such that $H_{1}(x) \leq F(x) \leq H_{2}(x)$ for each $x \in[c, d]$, and $H_{1}(x)=$ $H_{2}(x)=F(x)$ for each $x \in E$.

Moreover, let $[p, q] \subset[c, d]$ :

- If $p \in E$, then $H_{1}(q)-H_{1}(p) \leq F(q)-F(p)$ and $H_{2}(q)-H_{2}(p)=$ $\sup _{y \in[p, q]} F(y)-F(p)$.

- If $q \in E$, then $H_{1}(q)-H_{1}(p)=F(q)-\inf _{y \in[p, q]} F(y)$ and $H_{2}(q)-H_{2}(p) \leq$ $F(q)-F(p)$.

- If $F$ is continuous and $x_{o} \in E$, then both, $H_{1}$ and $H_{2}$ are continuous at $x_{0}$.

Proof. " $\Rightarrow$ " Let $H_{1}, H_{2}:[c, d] \rightarrow \mathbb{R}$,

$$
H_{1}(x)=\inf _{y \in[x, d]} F(y) \quad \text { and } \quad H_{2}(x)=\sup _{y \in[c, x]} F(y) .
$$

Clearly $H_{1}, H_{2}$ are increasing on $[c, d]$ and $H_{1}(x) \leq F(x) \leq H_{2}(x)$ for each $x \in[c, d]$ and $H_{1}(x)=H_{2}(x)=F(x)$ for each $x \in E$.

" $\Leftarrow$ " Let $x, y \in[c, d], x<y$. If $x \in E$, then $F(x)=H_{1}(x) \leq H_{1}(y) \leq F(y)$. If $y \in E$, then $F(y)=H_{2}(y) \geq H_{2}(x) \geq F(x)$. Thus $F$ is increasing* on $E$.

Corollary 4.1. [5, Proposition 2]. Let $F:[a, b] \rightarrow \mathbb{R}, E \subset[a, b], F$ increasing ${ }^{*}$ on $E$. Then $F$ is derivable a.e. on $E$. Moreover, if $F$ is $V B^{*}$ on $E$, then $F$ is derivable a.e. on $E$.

Corollary 4.2. Let $F:[a, b] \rightarrow \mathbb{R}, E \subset[a, b]$. If $F$ is increasing* on $E$ and $F$ is continuous at each point of $E$, then

$$
\mu_{F}^{*}(E)=m^{*}(F(E)) .
$$

Proof. Let for example $H_{1}:[a, b] \rightarrow \mathbb{R}$ be the function defined in Lemma 4.2. Then by Lemma 3.3 and Lemma 3.4 we obtain

$$
\mu_{F}^{*}(E)=\mu_{H_{1}}^{*}(E)=m^{*}\left(H_{1}(E)\right)=m^{*}(F(E)) .
$$

Lemma 4.3. Let $F:[a, b] \rightarrow \mathbb{R}$ and $E \subset[a, b]$ such that $\underline{D} F(x)>0$ for each $x \in E$. Then $F$ is increasing ${ }^{*} G$ on $E$. 
ProOF. Let

$$
E_{n}=\left\{x \in E: \frac{F(t)-F(x)}{t-x}>0, \quad 0<|t-x| \leq \frac{1}{n}\right\}, \quad n=1,2, \ldots .
$$

Let $E_{n i}=\left[\frac{i}{n}, \frac{i+1}{n}\right] \cap E_{n}, i=0, \pm 1, \pm 2, \ldots$. Then $E=\cup E_{n i}$ and $F$ is increasing* on each $E_{n i}$.

\section{The Denjoy-Lusin-Saks Theorem and an Extension of Two Theorems of Thomson}

Definition 5.1. $[5$, p. 415] Let $\omega, F:[a, b] \rightarrow \mathbb{R}, \omega$ strictly increasing on $[a, b]$. We define the lower and upper derivatives of $F$ with respect to $\omega$ at a point $x \in[a, b]$ as by

$$
\underline{D}_{\omega} F(x)=\liminf _{y \rightarrow x} \frac{F(y)-F(x)}{\omega(y)-\omega(x)} \quad \text { and } \quad \bar{D}_{\omega} F(x)=\limsup _{y \rightarrow x} \frac{F(y)-F(x)}{\omega(y)-\omega(x)} .
$$

$F$ is said to be derivable with respect to $\omega$ at $x$ if $\underline{D}_{\omega} F(x)=\bar{D}_{\omega} F(x) \in \mathbb{R}$. The derivative with respect to $\omega$ of $F$ at $x$ will be their common value and will be denoted by $F_{\omega}^{\prime}(x)$.

Definition 5.2. [5, p. 416] Let $F:[a, b] \rightarrow \mathbb{R}$. A set $E \subset[a, b]$ is said to be $F$-null if $E=C \cup N$, with $C$ an at most countable set and $\mu_{F}^{*}(N)=0$. If $F$ is the identity function, then the set $E$ is said to be $m$-null.

Lemma 5.1. Let $F:[a, b] \rightarrow \mathbb{R}, E \subset[a, b], c=\inf E, d=\sup E$. If $F$ is $V B^{*}$ on $E$, then there exists a strictly increasing function $H:[c, d] \rightarrow \mathbb{R}$ such that $\mu_{F}^{*}(A) \leq \mu_{H}^{*}(A)$, whenever $A \subset(c, d) \cap E$. Particularly, if $A \subseteq E$ is $H$-null, then $A$ is $F$-null.

Proof. By Lemma 4.1 there exist $F_{1}, F_{2}:[c, d] \rightarrow \mathbb{R}$ such that $F=F_{1}-F_{2}$ and $F_{1}, F_{2}$ are increasing* on $E$. Let $G:[c, d] \rightarrow \mathbb{R}, G=F_{1}+F_{2}$. Then $G$ is increasing* on $E$ and for $x, y \in[c, d]$ with $x<y$ and $\{x, y\} \cap E \neq \emptyset$ we have

$$
|F(y)-F(x)| \leq F_{1}(y)-F_{1}(x)+F_{2}(y)-F_{2}(x)=G(y)-G(x) .
$$

By Lemma 4.2 there exist two increasing functions $H_{1}, H_{2}:[c, d] \rightarrow \mathbb{R}$ such that $H_{1}(t) \leq G(t) \leq H_{2}(t)$ for $t \in[c, d]$ and $H_{1}(t)=H_{2}(t)=G(t)$ for $t \in E$. Let $H:[c, d] \rightarrow \mathbb{R}, H(t)=H_{1}(t)+H_{2}(t)+t$. If $x \in E$, then

$$
|F(y)-F(x)| \leq G(y)-G(x) \leq H_{2}(y)-H_{2}(x)<H(y)-H(x) .
$$


If $y \in E$, then

$$
|F(y)-F(x)| \leq G(y)-G(x) \leq H_{1}(y)-H_{1}(x)<H(y)-H(x) .
$$

Thus

$$
|F(y)-F(x)|<H(y)-H(x) .
$$

Let $A \subset(c, d) \cap E$. By (1) it follows immediately that $\mu_{F}^{*}(A) \leq \mu_{H}^{*}(A)$.

We show the second part. Let $D=\{x \in(c, d) \cap E: H$ is discontinuous at $x\}$. By (1), $F$ is continuous on $E \backslash D$. Thus, if $A \subseteq E$ is $H$-null, then $A$ is also $F$-null.

Lemma 5.2. Let $\omega, F:[a, b] \rightarrow \mathbb{R}, E \subset[a, b], \omega$ strictly increasing on $[a, b]$ and $F \in V B^{*}$ on $E$. Then there exists a set $A \subset \bar{E}$ such that $F$ is derivable with respect to $\omega$ on $\bar{E} \backslash A$, and $A$ is an $\omega$-null set.

Proof. Let $c=\inf E, d=\sup E$. Since $F \in V B^{*}$ on $E$, it follows that $F \in V B^{*}$ on $\bar{E}$ (see [12, p. 229]). We may suppose without loss of generality that $F$ is increasing* on $\bar{E}$ (see Lemma 4.1). Then this is [5, Proposition 4].

Lemma 5.3 (Faure). [5] Let $\omega, F:[a, b] \rightarrow \mathbb{R}, \omega$ strictly increasing. If $F_{\omega}^{\prime}(x)=0$ on $A \subset[a, b]$, then $\mu_{F}^{*}(A)=0$.

Lemma 5.4. Let $\omega, F:[a, b] \rightarrow \mathbb{R}, \omega$ strictly increasing, $E \subset[a, b]$. If $F \in V B^{*}$ on $E$, then the set $A=\left\{x \in E: \underline{D}_{\omega} F(x) \neq \bar{D}_{\omega} F(x)\right\}$ is F-null. Thus $F_{\omega}^{\prime}(x)$ exists (finite or infinite) on $E \backslash A$.

Proof. By Lemma 5.1, for $F$ there is a strictly increasing function $H$ : $[c, d] \rightarrow \mathbb{R}, c=\inf E, d=\sup E$, such that if $B \subseteq E$ is $H$-null, then $B$ is also $F$-null. Then the proof continues as in [5, Proposition 6].

Theorem 5.1. (An extension of Thomson's Theorems 44.1 and 44.2 of [13]). Let $\omega, F:[a, b] \rightarrow \mathbb{R}, \omega$ strictly increasing, and let $E \subset[a, b]$. If $F \in V B^{*} G$ on $E$, then $F_{\omega}^{\prime}(x)$ exists and is finite on $E$ except an $\omega$-null set $A$, and $F_{\omega}^{\prime}(x)$ exists (finite or infinite) on $E$ except a $F$-null subset $B$ of $A$.

Proof. The first part follows by Lemma 5.2. The second part follows by Lemma 5.4 and the fact that the union of countable many $\omega$-null sets is also an $\omega$-null set.

Lemma 5.5. Let $Z$ be a subset of $[a, b]$ such that $m^{*}(Z)=\mu_{F}^{*}(Z)=0$. Then $\Lambda(B(F ; Z))=0$. 
Proof. Note that $m^{*}(Z)=\mu_{\omega}^{*}(Z)$, where $\omega$ is the identity function. Let $\epsilon>0$. Since $m^{*}(Z)=\mu_{F}^{*}(Z)=0$, there exists $\delta: Z \rightarrow(0,+\infty)$ such that $V_{\delta}^{*}(\omega, Z)<\frac{\epsilon}{4}$ and $V_{\delta}^{*}(F, Z)<\frac{\epsilon}{4}$. By the covering lemma of [9, p. 143], there exists a sequence $\left\{\left(\left\langle x_{i}, y_{i}\right\rangle, x_{i}\right)\right\}_{i} \subset \beta_{\delta}^{*}(Z)$ such that $\left\{\left(\left\langle x_{i}, y_{i}\right\rangle, x_{i}\right)\right\}_{i=1}^{n}$ is a partition for all $n$ and $Z \subset \cup_{i}\left\langle x_{i}, y_{i}\right\rangle$. For each $i$ let $c_{i}=\inf F\left(\left\langle x_{i}, y_{i}\right\rangle\right)$ and $d_{i}=\sup F\left(\left\langle x_{i}, y_{i}\right\rangle\right)$. Then we have

$$
B(F, Z) \subset \cup_{i}\left(\left\langle x_{i}, y_{i}\right\rangle \times\left[c_{i}, d_{i}\right]\right) .
$$

For each $i$ let $z_{i} \in\left\langle x_{i}, y_{i}\right\rangle$ such that $d_{i}-c_{i}<3\left|F\left(z_{i}\right)-F\left(x_{i}\right)\right|$. Clearly

$$
\operatorname{diam}\left(\left\langle x_{i}, y_{i}\right\rangle \times\left[c_{i}, d_{i}\right]\right)<\left|y_{i}-x_{i}\right|+3\left|F\left(z_{i}\right)-F\left(x_{i}\right)\right|
$$

and

$$
\sum_{i} \operatorname{diam}\left(\left\langle x_{i}, y_{i}\right\rangle \times\left[c_{i}, d_{i}\right]\right) \leq V_{\delta}^{*}(\omega, Z)+3 V_{\delta}^{*}(F, Z)<\epsilon .
$$

It follows that $\Lambda(B(F, Z)) \leq \epsilon$, and $\Lambda(B(F, Z))=0$ since $\epsilon$ is arbitrary.

Remark 5.1. Lemma 5.5 is asserted by Faure in [5, p. 417] without proof.

Lemma 5.6. Let $F:[a, b] \rightarrow \mathbb{R}$, and let $Z$ be a subset of $[a, b]$ with $m^{*}(Z)=0$, such that $F \in V B^{*} G$ on $Z$. Then the following assertions are equivalent.

(i) $Z$ is F-null.

(ii) $\Lambda(B(F ; Z))=0$.

(iii) $m^{*}(F(Z))=0$.

Proof. (i) $\Rightarrow$ (ii) See Lemma 5.5 and note that $\Lambda(B(F ; A))=0$ whenever $A$ is a countable set.

(ii) $\Rightarrow$ (iii) This is evident (see for example [12, p. 269] or [6, p. 31]).

(iii) $\Rightarrow$ (i) Let $D=\{x \in Z: F$ is discontinuous at $x\}$. By [3, Theorem 8], it follows that $\mu_{F}^{*}(Z \backslash D)=0$. Thus $Z$ is $F$-null.

Corollary 5.1. Let $F:[a, b] \rightarrow \mathbb{R}$, and let $E$ be a subset of $[a, b]$ such that $F$ is $V B^{*} G$ on $E$. Let $Z=\left\{x \in E: F^{\prime}(x)\right.$ does not exist (finite or infinite) $\}$. Then:

(i) $F$ is derivable a.e. on $E$;

(ii) $Z$ is F-null; 
(iii) $\Lambda(B(F ; Z))=0$;

(iv) $m^{*}(F(Z))=0$.

Moreover, (ii), (iii) and (iv) are equivalent.

Proof. (i), (ii) follow from Theorem 5.1. The other parts follow by Lemma 5.6.

\section{Remark 5.2.}

Corollary 5.1, (i) is identic with Thomson's Theorem 44.1 of [13, p. 103].

Corollary 5.1, (ii) extends Thomson's Theorem 44.2 of [13, p. 104]. (Note that $F$ is not assumed to be continuous.)

Corollary 5.1, (i), (iii) is in fact Theorem A. Since for continuous functions, this result has been proved independently by Denjoy and Lusin [12], we call it the Denjoy-Lusin-Saks Theorem.

\section{The Denjoy-Young-Saks Theorem}

Theorem 6.1 (Denjoy-Young-Saks). ([5, Theorem 7] Let $\omega, F:[a, b] \rightarrow \mathbb{R}, \omega$ strictly increasing. Let

- $E_{1}=\{x: F$ is derivable with respect to $\omega\}$;

- $E_{2}=\left\{x: \underline{D}_{\omega} F(x)=-\infty\right.$ and $\left.\bar{D}_{\omega} F(x)=+\infty\right\}$;

- $E_{3}=\left\{x: \underline{D}_{\omega} F(x)=\bar{D}_{\omega} F(x)= \pm \infty\right\}$;

- $E_{4}=[a, b] \backslash\left(E_{1} \cup E_{2} \cup E_{3}\right)$.

Then

(i) $[a, b] \backslash\left(E_{1} \cup E_{2}\right)$ is $\omega$-null and contains $E_{3}$, so $E_{3}$ is $\omega$-null.

(ii) $E_{4}$ is both $\omega$-null and $F$-null.

Proof. The proof follows from Theorem 5.1 as in [5, p. 417].

Corollary 6.1. Let $F:[a, b] \rightarrow \mathbb{R}$. Let

- $E_{1}=\{x: F$ is derivable at $x\}$;

- $E_{2}=\{x: \underline{D} F(x)=-\infty$ and $\bar{D} F(x)=+\infty\}$;

- $E_{3}=\{x: \underline{D} F(x)=\bar{D} F(x)= \pm \infty\}$; 
- $E_{4}=[a, b] \backslash\left(E_{1} \cup E_{2} \cup E_{3}\right)$.

Then

(i) $[a, b] \backslash\left(E_{1} \cup E_{2}\right)$ is m-null and contains $E_{3}$, so $E_{3}$ is $m$-null;

(ii) $E_{4}$ is both m-null and F-null.

Moreover, (ii) may be replaced by " $\Lambda\left(B\left(F ; E_{4}\right)\right)=0$ ", or by " $m^{*}\left(F\left(E_{4}\right)\right)=0$ ".

Proof. (i) and (ii) follow by Theorem 6.1 with $\omega$ the identity function.

We show the second part. Since $E_{4} \subset[a, b] \backslash E_{2}$, it follows that $F$ is $V B^{*} G$ on $E_{4}$ (see [12, p. 234]). Since $E_{4}$ is $m$-null, the assertion follows by Lemma 5.6.

\section{Extensions of Theorem B and Theorem C of de la Vallée Poussin}

Theorem 7.1. Let $F: \mathbb{R} \rightarrow \mathbb{R}$, and let $E \subset \mathbb{R}$. If $F$ is increasing on $[a, b]$ and $F$ is constant on $(-\infty, a]$ and on $[b,+\infty)$, then $\mu_{F}^{*}(E)=F^{*}(E)$.

Proof. Let $D=\{x \in E: F$ is discontinuous at $x\}$. Then $D$ is countable. Suppose that $D=\left\{d_{1}, d_{2}, \ldots, d_{i}, \ldots\right\}$. By Lemma 2.1, (vi) and Lemma 3.1, (iv) we have

$$
F^{*}(D)=\sum_{i} F^{*}\left(\left\{d_{i}\right\}\right)=\sum_{i} \mu_{F}^{*}\left(\left\{d_{i}\right\}\right)=\mu_{F}^{*}(D) .
$$

The set $D$ being Borel measurable, by Lemma 2.1, (ii), (vii) and Lemma 3.1, (ii), (iii), it follows that

$$
F^{*}(E)=F^{*}(D)+F^{*}(E \backslash D)=\mu_{F}^{*}(D)+\mu_{F}^{*}(E \backslash D)=\mu_{F}^{*}(E) .
$$

Corollary 7.1. Let $F: \mathbb{R} \rightarrow \mathbb{R}$, and let $E \subset \mathbb{R}$. Suppose that $F$ is $V B$ on $[a, b]$ and $F$ is constant on $(-\infty, a]$ and on $[b,+\infty)$.

(i) $\mu_{F}^{*}(E)=\mu_{V_{F}}^{*}(E)=V_{F}^{*}(E)=\bar{V}_{F}^{*}(E)+\underline{V}_{F}^{*}(E)$;

(ii) If $\mu_{F}^{*}(E)=0$, then $V_{F}^{*}(E)=\bar{V}_{F}^{*}(E)=\underline{V}_{F}^{*}(E)=F^{*}(E)=\mu_{\bar{V}_{F}}^{*}(E)=$ $\mu_{\underline{V}_{F}}^{*}(E)=0$.

Proof. (i) follows from Lemma 3.1, (v), Theorem 7.1 and Lemma 2.2, and (ii) is evident. 
Corollary 7.2 (Theorem B). Let $F: \mathbb{R} \rightarrow \mathbb{R}, F \in V B$ on $[a, b], F$ constant on $(-\infty, a]$ and on $[b,+\infty)$. Let $Z=\{x \in[a, b]: F$ is continuous at $x$ and $F^{\prime}(x)$ does not exist (finite or infinite)\}. Then we have

$$
F^{*}(Z)=V_{F}^{*}(Z)=\mu_{F}^{*}(Z)=m^{*}(Z)=0=\Lambda(B(F ; Z))=0 .
$$

Proof. For $m^{*}(Z)=\mu_{F}^{*}(Z)=\Lambda(B(F ; Z))=0$ see Corollary 5.1, (i), (ii), (iii). That $V_{F}^{*}(Z)=F^{*}(Z)=0$ follows now by Corollary 7.1 .

Lemma 7.1. Let $F: \mathbb{R} \rightarrow \mathbb{R}, a \leq c<d \leq b$. Suppose that $F$ is constant on $(-\infty, a]$ and on $[b,+\infty)$. Then:

(i) $\bar{V}_{F}(d)-\bar{V}_{F}(c) \leq \bar{V}(F ;[c, d]) \leq V(F ;[c, d])=V_{F}(d)-V_{F}(c)$;

(ii) Let $E \subset[a, b]$ such that $[c, d] \subset[\inf E, \sup E]$. If $\left\{\left(c_{i}, d_{i}\right)\right\}_{i=1}^{\infty}$ are the intervals contiguous to $(\bar{E} \cap[c, d]) \cup\{c, d\}$ and $F$ is decreasing ${ }^{*}$ on $E$, then $\bar{V}(F ;[c, d]) \leq \sum_{i} V\left(F ;\left[c_{i}, d_{i}\right]\right)$, so $\bar{V}_{F}(d)-\bar{V}_{F}(c) \leq \sum_{i} V\left(F ;\left[c_{i}, d_{i}\right]\right)$.

Proof. (i) Let $\left\{\left[\alpha_{j}, \beta_{j}\right]\right\}_{j=1}^{n}$ be a finite set of nonoverlapping closed intervals contained in $[a, d]$. Suppose that $\alpha_{1}<\beta_{1} \leq \alpha_{2}<\beta_{2} \leq \ldots \leq \alpha_{n}<\beta_{n}$ and $c \in\left(\alpha_{j_{o}}, \beta_{j_{o}}\right)$ (the case $c \notin\left(\alpha_{j}, \beta_{j}\right), j=1,2, \ldots, n$ is easier). Then

$$
\begin{gathered}
\sum_{j=1}^{n}\left(F\left(\beta_{j}\right)-F\left(\alpha_{j}\right)\right)=\sum_{j=1}^{j_{o}-1}\left(F\left(\beta_{j}\right)-F\left(\alpha_{j}\right)\right)+F(c)-F\left(\alpha_{j_{o}}\right)+ \\
+F\left(\beta_{j_{o}}\right)-F(c)+\sum_{j=j_{o}+1}^{n}\left(F\left(\beta_{j}\right)-F\left(\alpha_{j}\right)\right) \leq \bar{V}_{F}(c)+\bar{V}(F ;[c, d]) .
\end{gathered}
$$

It follows that $\bar{V}_{F}(d)-\bar{V}_{F}(c) \leq \bar{V}(F ;[c, d])$. The other parts are evident.

(ii) Let $\left\{\left[a_{k}, b_{k}\right]\right\}_{k=1}^{m}$ be a finite set of nonoverlapping closed intervals contained in $[c, d]$. Clearly if $[\alpha, \beta] \cap E \neq \emptyset$ and $[\alpha, \beta] \subset[c, d]$, then $F(\beta)-F(\alpha) \leq$ 0 . Let

$$
\mathcal{A}=\left\{k \in\{1,2, \ldots, m\}: F\left(b_{k}\right)-F\left(a_{k}\right)>0\right\} .
$$

Then for each $k \in \mathcal{A},\left[a_{k}, b_{k}\right] \cap E=\emptyset$, so $\left[a_{k}, b_{k}\right] \subset\left[c_{i_{k}}, d_{i_{k}}\right]$ for some $i_{k}$. We also have that

$$
\sum_{k=1}^{m}\left(F\left(b_{k}\right)-F\left(a_{k}\right)\right) \leq \sum_{k \in \mathcal{A}}\left(F\left(b_{k}\right)-F\left(a_{k}\right)\right) \leq \sum_{i} V\left(f ;\left[c_{i}, d_{i}\right]\right) .
$$


Lemma 7.2. Let $F:[a, b] \rightarrow \mathbb{R}, F \in V B$ on $[a, b]$, and let $E \subset[a, b]$ such that $F$ is continuous at each point of $E$. If $F$ is decreasing* on $E$, then $\mu_{\bar{V}_{F}}^{*}(E)=0$. Consequently, if $F$ is decreasing $G$ on $E$, then $\mu_{\bar{V}_{F}}^{*}(E)=0$, and if $F$ is increasing $G$ on $E$, then $\mu_{\underline{V}_{F}}^{*}(E)=0$.

Proof. Let $c=\inf E, d=\sup E$, and let $\left\{\left(c_{i}, d_{i}\right)\right\}_{i=1}^{\infty}$ be the intervals contiguous to $\bar{E}$ (for $i=1,2, \ldots, n$ the proof is easier). It is well known that $V_{F}$ is continuous at each $x \in E$. Thus by Lemma 7.1 , (i), $\bar{V}_{F}$ is continuous at such a $x$. It follows that

$$
\mu_{\bar{V}_{F}}^{*}\left(E \cap\left(\cup_{i=1}^{\infty}\left\{c_{i}, d_{i}\right\} \cup\{c, d\}\right)\right)=0,
$$

so we may suppose without loss of generality that $E$ contains neither $c_{i}$ or $d_{i}$, nor $c$ or $d$. Since $\sum_{i=1}^{\infty} V\left(F ;\left[c_{i}, d_{i}\right]\right)<V(F ;[a, b])$, for $\epsilon>0$ there is an $i_{o}$ such that

$$
\sum_{i=i_{o}}^{\infty} V\left(F ;\left[c_{i}, d_{i}\right]\right)<\epsilon
$$

Let $G=(c, d) \backslash \cup_{i=1}^{i_{o}-1}\left[c_{i}, d_{i}\right]$. Clearly $E \subset G$. Let $\delta: E \rightarrow(0,+\infty)$ be such that $(x-\delta(x), x+\delta(x)) \subset G$. Let $\pi=\left\{\left(\left\langle x_{j}, y_{j}\right\rangle, x_{j}\right)\right\}_{j=1}^{p} \subset \beta_{\delta}^{*}(E)$ be a partition. We may suppose without loss of generality that $x_{j}<y_{j}$ for each $j=1,2, \ldots, p$. By Lemma 7.1 , we have that

$$
\sum_{j=1}^{p}\left(\bar{V}_{F}\left(y_{j}\right)-\bar{V}_{F}\left(x_{j}\right)\right) \leq \sum_{i=i_{o}}^{\infty} V\left(F ;\left[c_{i}, d_{i}\right]\right)<\epsilon .
$$

In general, it follows that $V_{\delta}^{*}\left(\bar{V}_{F} ; E\right) \leq 2 \epsilon$; so $\mu_{\bar{V}_{F}}^{*}(E) \leq 2 \epsilon$. Since $\epsilon$ is arbitrary, we obtain that $\mu_{\bar{V}_{F}}^{*}(E)=0$.

The second part follows from the fact that, if $F$ is increasing* $G$ on $E$, then $-F$ is decreasing ${ }^{*} G$ on $E$ and $\underline{V}_{F}(x)=\bar{V}_{-F}(x)$.

Corollary 7.3. Let $F:[a, b] \rightarrow \mathbb{R}$, be a $V B$ function, and let $E \subset[a, b]$ such that $F$ is continuous at each point of $E$. If $F$ is increasing* $G$ on $E$, then

$$
F^{*}(E)=V_{F}^{*}(E)=\mu_{F}^{*}(E)=\bar{V}_{F}^{*}(E) .
$$

Moreover, if $F$ is decreasing ${ }^{*} G$ on $E$, then

$$
-F^{*}(E)=V_{F}^{*}(E)=\mu_{F}^{*}(E)=\underline{V}_{F}^{*}(E) .
$$

Proof. See Lemma 7.2 and Corollary 7.1, (i). 
Theorem 7.2. Let $F:[a, b] \rightarrow \mathbb{R}$ be a $V B$ function. Let

$$
\begin{aligned}
& Z=\left\{x \in[a, b]: F^{\prime}(x) \text { does not exist (finite or infinite) }\right\} \\
& E_{+\infty}=\left\{x \in[a, b]: F^{\prime}(x)=+\infty\right\} \\
& E_{0}=\left\{x \in[a, b]: F^{\prime}(x)=0\right\} \\
& E_{-\infty}=\left\{x \in[a, b]: F^{\prime}(x)=-\infty\right\} \\
& P=\left\{x \in[a, b]: F^{\prime}(x) \in(0,+\infty)\right\} \\
& N=\left\{x \in[a, b]: F^{\prime}(x) \in(-\infty, 0)\right\}
\end{aligned}
$$

Then we have:

(i) $\mu_{\bar{V}_{F}}^{*}(Z)=\mu_{\bar{V}_{F}}^{*}\left(E_{0}\right)=\mu_{\bar{V}_{F}}^{*}\left(E_{-\infty}\right)=\mu_{\bar{V}_{F}}^{*}(N)=0$;

(ii) $\mu_{\underline{V}_{F}}^{*}(Z)=\mu_{\underline{V}_{F}}^{*}\left(E_{0}\right)=\mu_{\underline{V}_{F}}^{*}\left(E_{+\infty}\right)=\mu_{\underline{V}_{F}}^{*}(P)=0$;

(iii) $\mu_{\bar{V}_{F}}^{*}(E \cap P)=\mu_{V_{F}}^{*}(E \cap P)=V_{F}^{*}(E \cap P)=\mu_{F}^{*}(E \cap P)=(\mathcal{L}) \int_{E \cap P} F^{\prime}(t) d t$, whenever $E$ is a Lebesgue measurable subset of $[a, b]$;

(iv) $\mu_{\underline{V}_{F}}^{*}(E \cap N)=\mu_{V_{F}}^{*}(E \cap N)=V_{F}^{*}(E \cap N)=\mu_{F}^{*}(E \cap N)=-(\mathcal{L}) \int_{E \cap N} F^{\prime}(t) d t$, whenever $E$ is a Lebesgue measurable subset of $[a, b]$;

(v) $\bar{V}_{F}^{*}(E)=\mu_{\bar{V}_{F}}^{*}(E)=\mu_{\bar{V}_{F}}^{*}\left(E \cap E_{+\infty}\right)+(\mathcal{L}) \int_{E \cap P} F^{\prime}(t) d t$, whenever $E$ is a Lebesgue measurable subset of $[a, b]$ and $F$ is continuous at each point of $E$;

(vi) $\underline{V}_{F}^{*}(E)=\mu_{\underline{V}_{F}}^{*}(E)=\mu_{\underline{V}_{F}}^{*}\left(E \cap E_{-\infty}\right)+(\mathcal{L}) \int_{E \cap N} F^{\prime}(t) d t$, whenever $E$ is a Lebesgue measurable subset of $[a, b]$ and $F$ is continuous at each point of $E$;

(vii) $F^{*}(E)=F^{*}\left(E \cap E_{+\infty}\right)+F^{*}\left(E \cap E_{-\infty}\right)+(\mathcal{L}) \int_{E} F^{\prime}(t) d t$, whenever $E$ is a Lebesgue measurable subset of $[a, b]$ and $F$ is continuous at each point of $E$;

(viii) $V_{F}^{*}(E)=F^{*}\left(E \cap E_{+\infty}\right)+\left|F^{*}\left(E \cap E_{-\infty}\right)\right|+(\mathcal{L}) \int_{E}\left|F^{\prime}(t)\right| d t$, whenever $E$ is a Lebesgue measurable subset of $[a, b]$ and $F$ is continuous at each point of $E$;

(ix) $V_{F}^{*}([a, b] \backslash A)=m^{*}([a, b] \backslash A)=0$, where $A=\left\{x \in[a, b]: V_{F}^{\prime}(x)=\right.$ $\left|F^{\prime}(x)\right|, F$ is continuous at $\left.x\right\}$. 
Proof. Note that $F$ satisfies Lusin's condition $(N)$ on $E_{0} \cup P \cup N$ (see [12]).

(i) By Corollary $7.2, \mu_{F}^{*}(Z)=0$, and by Lemma $5.3, \mu_{F}^{*}\left(E_{0}\right)=0$. It follows that $\mu_{\bar{V}_{F}}^{*}(Z)=\mu_{F}^{*}\left(E_{0}\right)=0$ (see Corollary 7.1, (ii)). By Lemma 4.3, $F$ is decreasing ${ }^{*} G$ on $E_{-\infty} \cup N$ so by Lemma 7.2 we have that $\mu_{V_{F}}^{*}\left(E_{-\infty}\right)=$ $\mu_{\bar{V}_{F}}^{*}(N)=0$.

(ii) The proof follows by (i), because $\mu_{\underline{V}_{F}}^{*}=\mu_{\bar{V}_{-F}}^{*}$.

(iii) By Lemma 3.2 we have $\mu_{F}^{*}(E \cap P)=(\mathcal{L}) \int_{E \cap P} F^{\prime}(t) d t$, and by Corollary 7.1, (i) it follows that

$$
\mu_{F}^{*}(E \cap P)=\mu_{V_{F}}^{*}(E \cap P)=\mu_{\bar{V}_{F}}^{*}(E \cap P)+\mu_{\underline{V}_{F}}^{*}(E \cap P)=\mu_{\bar{V}_{F}}^{*}(E \cap P)
$$

(see also (ii)).

(iv) The proof is similar to that of (iii).

(v) That $\bar{V}_{F}^{*}(E)=\mu_{\bar{V}_{F}}^{*}(E)$ follows by Theorem 7.1. Since $Z \cup E_{+\infty} \cup E_{0} \cup$ $E_{-\infty} \cup P \cup N=[a, b]$ and because $Z, E_{+\infty}, E_{0}, E_{-\infty}, P$ and $N$ are all Borel sets (so $\mu_{\bar{V}_{F}}^{*}$-measurable), Lemma 3.1, (ii) and by (i) and (iii) above, it follows that

$$
\begin{aligned}
\mu_{\bar{V}_{F}}^{*}(E)= & \mu_{\bar{V}_{F}}^{*}(E \cap Z)+\mu_{\bar{V}_{F}}^{*}\left(E \cap E_{+\infty}\right)+\mu_{\bar{V}_{F}}^{*}\left(E \cap E_{0}\right) \\
& +\mu_{\bar{V}_{F}}^{*}\left(E \cap E_{-\infty}\right)+\mu_{\bar{V}_{F}}^{*}(E \cap P)+\mu_{\bar{V}_{F}}^{*}(E \cap N) \\
= & \mu_{\bar{V}_{F}}^{*}\left(E \cap E_{+\infty}\right)+\mu_{\bar{V}_{F}}^{*}(E \cap P) \\
= & \mu_{\bar{V}_{F}}^{*}\left(E \cap E_{+\infty}\right)+(\mathcal{L}) \int_{E \cap P} F^{\prime}(t) d t .
\end{aligned}
$$

(vi) The proof is similar to that of (v).

(vii) We have

$$
\begin{aligned}
F^{*}(E)= & \bar{V}_{F}^{*}(E)-\underline{V}_{F}^{*}(E)=\bar{V}_{F}^{*}\left(E \cap E_{+\infty}\right) \\
& +(\mathcal{L}) \int_{E \cap P} F^{\prime}(t) d t-\underline{V}_{F}^{*}\left(E \cap E_{-\infty}\right)+(\mathcal{L}) \int_{E \cap N} F^{\prime}(t) d t \\
= & F^{*}\left(E \cap E_{+\infty}\right)+F^{*}\left(E \cap E_{-\infty}\right)+(\mathcal{L}) \int_{E \cap\left(P \cup N \cup E_{0}\right)} F^{\prime}(t) d t \\
= & F^{*}\left(E \cap E_{+\infty}\right)+F^{*}\left(E \cap E_{-\infty}\right)+(\mathcal{L}) \int_{E} F^{\prime}(t) d t
\end{aligned}
$$

(see (ii), (i) and the facts that $(\mathcal{L}) \int_{E_{0}} F^{\prime}(t) d t=0$ and $\left.m\left(E \backslash\left(P \cup N \cup E_{0}\right)\right)=0\right)$. 
(viii) By Corollary 7.1, (i) we have:

$$
\begin{aligned}
V_{F}^{*}(E)= & \bar{V}_{F}^{*}(E)+\underline{V}_{F}^{*}(E)=\bar{V}_{F}^{*}\left(E \cap E_{+\infty}\right) \\
& +(\mathcal{L}) \int_{E \cap P} F^{\prime}(t) d t+\underline{V}_{F}^{*}\left(E \cap E_{-\infty}\right)+(\mathcal{L}) \int_{E \cap N}\left|F^{\prime}(t)\right| d t \\
= & F^{*}\left(E \cap E_{+\infty}\right)+\left|F^{*}\left(E \cap E_{-\infty}\right)\right|+(\mathcal{L}) \int_{E}\left|F^{\prime}(t)\right| d t
\end{aligned}
$$

(see (ii) and (i)).

(ix) By $\left[11\right.$, Theorem, p. 15] it follows that $V_{F}^{\prime}(x)=\left|F^{\prime}(x)\right| \in[0,+\infty)$ a.e. on $[a, b]$, so $m^{*}([a, b] \backslash A)=0$. By (viii), we have

$$
V_{F}^{*}([a, b] \backslash A)=F^{*}\left(([a, b] \backslash A) \cap E_{+\infty}\right)+F^{*}\left(([a, b] \backslash A) \cap E_{-\infty}\right) .
$$

If $x \in E_{+\infty}$, then $F^{\prime}(x)=+\infty$, so $V_{F}^{\prime}(x)=+\infty$. Hence $x \in A$, and so $([a, b] \backslash A) \cap E_{+\infty}=\emptyset$. Similarly $([a, b] \backslash A) \cap E_{-\infty}=\emptyset$. It follows that $V_{F}^{*}([a, b] \backslash A)=0$.

Remark 7.1. Theorem 7.2, (vii), (viii), (ix) strictly contains Theorem C, because in (vii) and (viii) the set $E$ is not only Borel but also Lebesgue measurable. Note also in order to prove Theorem C, Saks uses the Lebesgue Decomposition Theorem [12, p. 119], whereas our proof does not use this decomposition; it is instead essentially based on Theorem 8.2 of [4] (see Lemma 3.2).

\section{A de la Vallée Poussin Type Theorem for VB*G Functions (An Extension of a Theorem of Thomson)}

Lemma 8.1 (Thomson). [13, Lemma 42.1]. Let $F:[a, b] \rightarrow \mathbb{R}, E \subset[a, b]$. Then $\mu_{F}^{*}\left(E_{o}\right)=0$, where $E_{o}=\left\{x \in[a, b]: F^{\prime}(x)=0\right\}$.

Definition 8.1. With the notations of Definition 3.1, let:

- $\bar{V}_{\delta}^{*}(F ; E)=\sup \left\{\sum_{i=1}^{n} \Delta F^{+}\left(\left\langle x_{i}, y_{i}\right\rangle\right): \pi=\left\{\left(\left\langle x_{i}, y_{i}\right\rangle, x_{i}\right)\right\}_{i=1}^{n}\right.$ is a partition, $\left.\pi \subset \beta_{\delta}^{*}(E)\right\}$;

- $\underline{V}_{\delta}^{*}(F ; E)=\sup \left\{\sum_{i=1}^{n} \Delta F^{-}\left(\left\langle x_{i}, y_{i}\right\rangle\right): \pi=\left\{\left(\left\langle x_{i}, y_{i}\right\rangle, x_{i}\right)\right\}_{i=1}^{n}\right.$ is a partition, $\left.\pi \subset \beta_{\delta}^{*}(E)\right\}$;

- $\bar{\mu}_{F}^{*}(E)=\inf _{\delta} \bar{V}_{\delta}^{*}(F ; E)$;

- $\underline{\mu}_{F}^{*}(E)=\inf _{\delta} \underline{V}_{\delta}^{*}(F ; E)$; 
Lemma 8.2. Let $F: \mathbb{R} \rightarrow \mathbb{R}, E \subset \mathbb{R}$. Then we have:

(i) $\bar{\mu}_{F}^{*}(E) \leq \mu_{F}^{*}(E)$;

(ii) $\underline{\mu}_{F}^{*}(E) \leq \mu_{F}^{*}(E)$;

(iii) $\mu_{F}^{*}(E) \leq \bar{\mu}_{F}^{*}(E)+\underline{\mu}_{F}^{*}(E)$.

Proof. All assertions follow from the equality

$$
|F(y)-F(x)|=\Delta F^{+}([x, y])+\Delta F^{-}([x, y]) .
$$

Lemma 8.3. Let $F:[a, b] \rightarrow \mathbb{R}$. Then $\mu_{F}^{*}(E)=\bar{\mu}_{F}^{*}(E)$ whenever $E \subset\{x \in$ $\left.[a, b]: F^{\prime}(x) \in[0,+\infty]\right\}$.

Proof. We always have $\mu_{F}^{*}(E) \geq \bar{\mu}_{F}^{*}(E)$. We show the converse inequality. Let $P=\left\{x \in[a, b]: F^{\prime}(x) \in(0,+\infty]\right\}, A \subset P$ and let $\eta: A \rightarrow(0,+\infty)$ such that

$$
\frac{F(y)-F(x)}{y-x}>0 \text { whenever } y \in(x-\eta(x), x+\eta(x)) \backslash\{x\} .
$$

Let $\delta: A \rightarrow(0,+\infty)$, and let $\delta_{1}(x)=\min \{\delta(x), \eta(x)\}$ for each $x \in A$. If $([x, y], x)$ or $([x, y], y) \in \beta_{\delta_{1}}^{*}(A)$, then $0<F(y)-F(x)=\Delta F^{+}([x, y])$. It follows that $(\langle x, y\rangle, x) \in \beta_{\delta_{1}}^{*}(A)$ and $\Delta F^{+}(\langle x, y\rangle)=|F(y)-F(x)|$. Hence

$$
\mu_{F}^{*}(A) \leq V_{\delta_{1}}^{*}(F ; A)=\bar{V}_{\delta_{1}}^{*}(F ; A) \leq \bar{V}_{\delta}^{*}(F ; A) .
$$

Therefore $\mu_{F}^{*}(A) \leq \bar{\mu}_{F}^{*}(A)$. Now we obtain

$$
\mu_{F}^{*}(E) \leq \mu_{F}^{*}(E \cap P)+\mu_{F}^{*}\left(E \cap E_{0}\right)=\mu_{F}^{*}(E \cap P) \leq \bar{\mu}_{F}^{*}(E \cap P) \leq \bar{\mu}_{F}^{*}(E),
$$

where $E_{0}=\left\{x \in[a, b]: F^{\prime}(x)=0\right\}$.

Lemma 8.4. Let $F:[a, b] \rightarrow \mathbb{R}$. Then $\bar{\mu}_{F}^{*}(E)=0$ whenever $E \subset\{x \in[a, b]$ : $\left.F^{\prime}(x) \in[-\infty, 0]\right\}$.

Proof. Let $N=\left\{x \in[a, b]: F^{\prime}(x) \in[-\infty, 0)\right\}, A \subset N$, and let $\delta: A \rightarrow$ $(0,+\infty)$ such that

$$
\frac{F(y)-F(x)}{y-x}<0 \text { whenever } y \in(x-\delta(x), x+\delta(x)) \backslash\{x\} .
$$

If $([x, y], x)$ or $([x, y], y) \in \beta_{\delta}^{*}(A)$, then $F(y)-F(x)<0$; so $\Delta F^{+}([x, y])=0$. It follows that $\bar{\mu}_{F}^{*}(A) \leq \bar{V}_{\delta}^{*}(F ; A)=0$, so $\bar{\mu}_{F}^{*}(A)=0$. Now we obtain that

$$
\bar{\mu}_{F}^{*}(E) \leq \bar{\mu}_{F}^{*}(E \cap N)+\bar{\mu}_{F}^{*}\left(E \cap E_{0}\right) \leq 0+\mu_{F}^{*}\left(E \cap E_{0}\right)=0+0=0,
$$

where $E_{0}=\left\{x \in[a, b]: F^{\prime}(x)=0\right\}$. 
Thomson's Variational Measure and Some Classical Theorems 541

Theorem 8.1. (An extension of Theorem 46.3 of [13, p. 107]).

Let $F: \mathbb{R} \rightarrow \mathbb{R}$, and let $E \subset \mathbb{R}$ such that $F$ is continuous at each point of $E$ and $F \in V B^{*} G$ on $E$. Let $E_{+\infty}=\left\{x: F^{\prime}(x)=+\infty\right\}, E_{-\infty}=\{x$ : $\left.F^{\prime}(x)=-\infty\right\}, D=\left\{x: F^{\prime}(x) \in(-\infty,+\infty)\right\}, P=\left\{x: F^{\prime}(x)=(0,+\infty)\right\}$, $N=\left\{x: F^{\prime}(x)=(-\infty, 0)\right\}$. Then we have:

(i) $\mu_{F}^{*}(E)=\mu_{F}^{*}\left(E \cap E_{+\infty}\right)+\mu_{F}^{*}\left(E \cap E_{-\infty}\right)+\mu_{F}^{*}(E \cap P)+\mu_{F}^{*}(E \cap N)=$ $\mu_{F}^{*}\left(E \cap E_{+\infty}\right)+\mu_{F}^{*}\left(E \cap E_{-\infty}\right)+\mu_{F}^{*}(E \cap D)$;

(ii) $\bar{\mu}_{F}^{*}(E)=\mu_{F}^{*}\left(E \cap E_{+\infty}\right)+\mu_{F}^{*}(E \cap P)$;

(iii) $\underline{\mu}_{F}^{*}(E)=\mu_{F}^{*}\left(E \cap E_{-\infty}\right)+\mu_{F}^{*}(E \cap N)$.

Therefore $\mu_{F}^{*}(E)=\bar{\mu}_{F}^{*}(E)+\underline{\mu}_{F}^{*}(E)$.

Moreover, if $E$ is Lebesgue measurable, then

(iv) $\mu_{F}^{*}(E)=\mu_{F}^{*}\left(E \cap E_{+\infty}\right)+\mu_{F}^{*}\left(E \cap E_{-\infty}\right)+(\mathcal{L}) \int_{E \cap D}\left|F^{\prime}(t)\right| d t$;

(v) $\bar{\mu}_{F}^{*}(E)=\mu_{F}^{*}\left(E \cap E_{+\infty}\right)+(\mathcal{L}) \int_{E \cap P} F^{\prime}(t) d t$;

(vi) $\underline{\mu}_{F}^{*}(E)=\mu_{F}^{*}\left(E \cap E_{-\infty}\right)-(\mathcal{L}) \int_{E \cap N} F^{\prime}(t) d t$,

Proof. Let $E_{0}=\left\{x \in E: F^{\prime}(x)=0\right\}$ and $Z=\left\{x \in E: F^{\prime}(x)\right.$ does not exist (finite or infinite) $\}$. The sets $Z, E_{0}, E_{+\infty}, E_{-\infty}, D, P, N$ are all Borel (see Hajek's Theorem of $[1$, p. 57]).

(i) Since $Z \cup E_{+\infty} \cup E_{+\infty} \cup D=\mathbb{R}$, we obtain

$$
\begin{aligned}
\mu_{F}^{*}(E) & =\mu_{F}^{*}(E \cap Z)+\mu_{F}^{*}\left(E \cap E_{+\infty}\right)+\mu_{F}^{*}\left(E \cap E_{-\infty}\right)+\mu_{F}^{*}(E \cap D) \\
& =\mu_{F}^{*}\left(E \cap E_{+\infty}\right)+\mu_{F}^{*}\left(E \cap E_{-\infty}\right)+\mu_{F}^{*}(E \cap D)
\end{aligned}
$$

by Lemma 3.1, (ii), and Corollary 5.1, (ii). Since $D=E_{o} \cup P \cup N$, we obtain

$$
\begin{aligned}
\mu_{F}^{*}(E \cap D) & =\mu_{F}^{*}\left(E \cap E_{o}\right)+\mu_{F}^{*}(E \cap P)+\mu_{F}^{*}(E \cap N) \\
& =\mu_{F}^{*}(E \cap P)+\mu_{F}^{*}(E \cap N)
\end{aligned}
$$

by Lemma 3.1, (ii), and Lemma 8.1.

(ii) ${ }^{1}$ Since $Z \cup E_{+\infty} \cup P \cup\left(E_{o} \cup N \cup E_{-\infty}\right)=\mathbb{R}$, we obtain

$$
\begin{aligned}
\bar{\mu}_{F}^{*}(E) & =\bar{\mu}_{F}^{*}(E \cap Z)+\bar{\mu}_{F}^{*}\left(E \cap E_{+\infty}\right)+\bar{\mu}_{F}^{*}(E \cap P)+\bar{\mu}_{F}^{*}\left(E \cap\left(E_{o} \cup N \cup E_{-\infty}\right)\right) \\
& =\bar{\mu}_{F}^{*}(E \cap Z)+\bar{\mu}_{F}^{*}\left(E \cap E_{+\infty}\right)+\bar{\mu}_{F}^{*}(E \cap P)
\end{aligned}
$$

by Lemma 3.1, (ii), Lemma 8.3 and Lemma 8.4. And we have

$$
0 \leq \bar{\mu}_{F}^{*}(E \cap Z) \leq \mu_{F}^{*}(E \cap Z)=0
$$

\footnotetext{
${ }^{1}$ The proof of Theorem 8.1, (ii) uses that $\bar{\mu}_{F}^{*}$ is a metric outer measure.
} 
by Lemma 8.2 , (i), and Corollary 5.1, (ii).

(iii) The proof is similar to that of (ii).

(iv), (v) and (vi) follow by Lemma 3.2.

\section{Characterizations of $\mathrm{VB}^{*} \mathrm{G} \cap(\mathrm{N})$ for Lebesgue Measurable Functions}

Corollary 9.1. Let $F:[a, b] \rightarrow \mathbb{R}$ and let $E$ be a Lebesgue measurable subset of $[a, b]$. The following assertions are equivalent.

(i) $F \in V B^{*} G \cap(N)$ on $E$.

(ii) $F \in V B^{*} G \cap(N)$ on $Z$, whenever $Z$ is a null subset of $E$.

(iii) There exists a countable subset $E_{1}$ of $E$ such that $\mu_{F}^{*}(Z)=0$, whenever $Z$ is a null subset of $E \backslash E_{1}$.

(iv) $Z$ is $F$-null whenever $Z$ is a null subset of $E$.

Proof. For (i) $\Leftrightarrow$ (ii) $\Leftrightarrow$ (iii) see [3, Theorem 9] and (iii) $\Rightarrow$ (iv) is evident.

(iv) $\Rightarrow$ (ii) Let $Z$ be a null subset of $E$. Then $Z$ is $F$-null, so by Lemma 5.6, $m(F(Z))=0$. It follows that $F \in(N)$ on $Z$. For $Z$ there is a countable set $D$ such that $\mu_{F}^{*}(Z \backslash D)=0$. By [13, Theorem 40.1], $F$ is $V B^{*} G$ on $Z \backslash D$, so on $Z$.

\section{A Characterization of $\mathrm{VB}^{*} \mathrm{G} \cap \mathrm{N}^{+\infty}$ on a Lebesgue Measurable Set}

Definition 10.1 (Saks). [2, p. 79] Let $F: \mathbb{R} \rightarrow \mathbb{R} . F$ is said to be $N^{+\infty}$ on a real set $E$ if the set $\left(\left\{x \in E:\left(F_{\mid E}\right)^{\prime}(x)=+\infty\right\}\right)$ is of Lebesgue measure zero.

Lemma 10.1. Let $F: \mathbb{R} \rightarrow \mathbb{R}$, and let $E \subset \mathbb{R}$ such that $F$ is $V B^{*} G$ on $E$. Let $E_{+\infty}=\left\{x: F^{\prime}(x)=+\infty\right\}$. Then the following assertions are equivalent.

(i) $F$ is $N^{+\infty}$ on $E$.

(ii) $m^{*}\left(F\left(E \cap E_{+\infty}\right)\right)=0$.

Proof. (i) $\Rightarrow$ (ii) Let $E_{1}=\{x \in E: x$ is an accumulation point for $E\}$. Then $E \backslash E_{1}$ is at most countable and $E_{1} \cap E_{+\infty} \subset\left\{x \in E:\left(F_{\mid E}\right)^{\prime}(x)=+\infty\right\}$.

(ii) $\Rightarrow$ (i) Let $Z=\left\{x \in E: F^{\prime}(x)\right.$ does not exist (finite or infinite) $\}$. Then we have $\left\{x \in E:\left(F_{\mid E}\right)^{\prime}(x)=+\infty\right\} \subset Z \cup E_{+\infty}$, and (i) follows because $m^{*}(F(E \cap Z))=0$ by Corollary 5.1 , (iv). 
Lemma 10.2. Let $F: \mathbb{R} \rightarrow \mathbb{R}$ and $E \subset \mathbb{R}$. If $\bar{\mu}_{F}^{*}(E)<+\infty$, then $F \in V B^{*} G$ on $E$.

Proof. Suppose that $\bar{\mu}_{F}^{*}(E)=M<+\infty$. For $\epsilon=1$ there is a $\delta: E \rightarrow$ $(0,+\infty)$ such that $\bar{V}_{\delta}^{*}(F ; E)<M+1$. Let

$$
E_{n}=\left\{x: \delta(x)>\frac{1}{n}\right\} \quad \text { and } \quad E_{n i}=E_{n} \cap\left[\frac{i}{n}, \frac{i+1}{n}\right], \quad i=0, \pm 1, \pm 2, \ldots .
$$

If $E_{n i}$ is countable, then $F$ is $V B^{*} G$ on this set. Fix some uncountable set $E_{n i}$ and let $c_{n i}=\inf E_{n i}, d_{n i}=\sup E_{n i}$. We show that $F \in \overline{V B}\left(E_{n i} ;\left[c_{n i}, d_{n i}\right]\right)$ (for the definition see [2, Definition 2.7.1]). Let $\left\{\left[c_{k}, d_{k}\right]\right\}_{k=1}^{p}$ be a finite set of nonoverlapping closed intervals such that $\left\{c_{k}, d_{k}\right\} \cap E_{n i} \neq \emptyset$. Clearly, if $c_{k} \in$ $E_{n i}$, then $\left(\left[c_{k}, d_{k}\right], c_{k}\right) \in \beta_{\delta}^{*}(E)$, and if $d_{k} \in E_{n i}$, then $\left(\left[c_{k}, d_{k}\right], d_{k}\right) \in \beta_{\delta}^{*}(E)$. It follows that

$$
\sum_{k=1}^{p}\left(F\left(d_{k}\right)-F\left(c_{k}\right)\right) \leq \sum_{k=1}^{p} \Delta F^{+}\left(\left[c_{k}, d_{k}\right]\right)<\bar{V}_{\delta}^{*}(F ; E)<M+1 .
$$

Thus $F \in \overline{V B}\left(E_{n i} ;\left[c_{n i}, d_{n i}\right]\right)$. By $[2$, Theorem 2.8.1, (xii), (i)], we obtain that $F \in V B^{*}$ on $E_{n i}$; so $F \in V B^{*} G$ on $E$.

Theorem 10.1. Let $F: \mathbb{R} \rightarrow \mathbb{R}$ and let $E$ be a Lebesgue measurable subset of $\mathbb{R}$. Let $E_{+\infty}=\left\{x: F^{\prime}(x)=+\infty\right\}$. Then the following assertions are equivalent.

(i) $F \in V B^{*} G \cap N^{+\infty}$ on $E$.

(ii) there exists a countable subset $E_{1}$ of $E$ such that $\bar{\mu}_{F}^{*}(Z)=0$ whenever $Z \subset E \backslash E_{1}$ and $m^{*}(Z)=0$.

Proof. (i) $\Rightarrow$ (ii) Since $F$ is $V B^{*} G$ on $E$, there exists a countable set $E_{1}$ such that $F$ is continuous at each point of $E \backslash E_{1}$ (see [12]). Let $Z \subset E \backslash E_{1}$ with $m^{*}(Z)=0$. Then we have

$$
\bar{\mu}_{F}^{*}(Z)=\mu_{F}^{*}\left(Z \cap E_{+\infty}\right)=m^{*}\left(F\left(Z \cap E_{+\infty}\right)\right)=0
$$

by Theorem 8.1, (v), Lemma 5.6, (i), (iii) and Lemma 10.1.

(ii) $\Rightarrow$ (i) By Corollary 5.1, (i), $m^{*}\left(E_{+\infty}\right)=0$, and by Lemma 8.3, we obtain that

$$
\mu_{F}^{*}\left(\left(E \cap E_{+\infty}\right) \backslash E_{1}\right)=\bar{\mu}_{F}^{*}\left(\left(E \cap E_{+\infty}\right) \backslash E_{1}\right)=0 .
$$

It follows that $m^{*}\left(F\left(E \cap E_{+\infty}\right)\right)=0$ (see Lemma 3.1, (vi)); so $F$ is $N^{+\infty}$ on $E$ (see Lemma 10.1). Let $Z \subset E \backslash E_{1}$ with $m^{*}(Z)=0$. Since $\bar{\mu}_{F}^{*}(Z)=0$, by Lemma 10.2, it follows that $F \in V B^{*} G$ on $Z$. Hence $F \in V B^{*} G$ on $E \backslash E_{1}$, so on $E$ (see [3, Theorem 1]). 
Lemma 10.3. Let $F, G: \mathbb{R} \rightarrow \mathbb{R}, E \subset \mathbb{R}, \alpha, \beta \geq 0$. Then

$$
\bar{\mu}_{\alpha F+\beta G}^{*}(E) \leq \alpha \cdot \bar{\mu}_{F}^{*}(E)+\beta \cdot \bar{\mu}_{G}^{*}(E) .
$$

Proof. From $\Delta(\alpha F+\beta G)^{+}([x, y]) \leq \alpha \cdot \Delta F^{+}([x, y])+\beta \cdot \Delta G^{+}([x, y])$ it follows immediately that $\bar{\mu}_{\alpha F+\beta G}^{*}(E) \leq \alpha \cdot \bar{\mu}_{F}^{*}(E)+\beta \cdot \bar{\mu}_{G}^{*}(E)$.

Corollary 10.1. Let $E \subset \mathbb{R}$ be a Lebesgue measurable set. Let

$$
\mathcal{A}=\left\{F: \mathbb{R} \rightarrow \mathbb{R}: F \in V B^{*} G \cap N^{+\infty} \text { on } E\right\} .
$$

Then $\mathcal{A}$ is a semi-linear subspace, i.e., $\alpha_{1} F_{1}+\alpha_{2} F_{2} \in \mathcal{A}$, whenever $\alpha_{1}, \alpha_{2} \geq 0$ and $F_{1}, F_{2} \in \mathcal{A}$.

Proof. Let $\alpha_{1}, \alpha_{2} \geq 0$ and $F_{1}, F_{2} \in \mathcal{A}$. Clearly $\alpha_{1} F_{1}+\alpha_{2} F_{2} \in V B^{*} G$. By Theorem 10.1, there exist two countable subsets $E_{1}, E_{2}$ of $E$ such that $\bar{\mu}_{F}^{*}\left(Z_{1}\right)=0$ whenever $Z_{1}=E \backslash E_{1}$ and $m^{*}\left(Z_{1}\right)=0$, and $\bar{\mu}_{F_{2}}^{*}\left(Z_{2}\right)=0$ whenever $Z_{2} \subset E \backslash E_{2}$ and $m^{*}\left(Z_{2}\right)=0$. Let $Z \subset E \backslash\left(E_{1} \cup E_{2}\right)$ with $m^{*}(Z)=0$. Then $\bar{\mu}_{F_{1}}^{*}(Z)=\bar{\mu}_{F_{2}}^{*}(Z)=0$. By Lemma $10.3, \bar{\mu}_{\alpha_{1} F_{1}+\alpha_{2} F_{2}}^{*}(Z)=0$; so by Theorem 10.1 we obtain that $\alpha_{1} F_{1}+\alpha_{2} F_{2} \in \mathcal{A}$.

Corollary 10.2. Let $E \subset \mathbb{R}$ be a Lebesgue measurable set and let

$\mathcal{A}_{1}=\left\{F: \mathbb{R} \rightarrow \mathbb{R}: F \in V B^{*} G\right.$ on $E$ and $\left.m\left(F\left(E \cap\left\{x: F^{\prime}(x)= \pm \infty\right\}\right)\right)=0\right\}$.

Then $\mathcal{A}_{1}$ is a linear space.

Proof. Let $\mathcal{A}$ be defined as in Corollary 10.1. If $F \in \mathcal{A}_{1}$, then $F$ and $-F$ belong to $\mathcal{A}$. Applying Corollary 10.1 and Lemma 10.1, it follows that $\mathcal{A}_{1}$ is a linear space.

Remark 10.1. Note that $\mathcal{A}_{1}=\left\{F: \mathbb{R} \rightarrow \mathbb{R}: F \in V B^{*} G \cap(N)\right.$ on $\left.E\right\}$. This follows by Lemma 5.6 and the well known fact that $F \in(N)$ on the set $\left\{x \in E: F^{\prime}(x)\right.$ exists and is finite $\}$. Therefore Corollary 10.2 is a special case of [3, Corollary 3].

\section{References}

[1] A. M. Bruckner, Differentiation of real functions, Lect. Notes in Math., vol. 659, Springer-Verlag, 1978.

[2] V. Ene, Real functions - current topics, Lect. Notes in Math., vol. 1603, Springer-Verlag, 1995. 
Thomson's Variational Measure and Some Classical Theorems 545

[3] V. Ene, Characterizations of $V B^{*} G \cap(N)$, Real Analysis Exchange 23 (1997/8), no. 2, 571-600.

[4] V. Ene, Thomson's variational measure, Real Analysis Exchange 24 (1998/9), no. 2, 523-566.

[5] C. A. Faure, Sur le théorème de Denjoy-Young-Saks, C. R. Acad. Sci. Paris 320 (1995), no. Série I, 415-418.

[6] J. Foran, On functions whose graph is of linear measure 0 on sets of measure 0, Fund Math. XCVI (1977), 31-36.

[7] K. Krzyzewski, On change of variable in the Denjoy-Perron integral (I),(II), Coll. Math. 9 (1962), 99-104 and 317-323.

[8] H. Lebesgue, Leçons sur l'intégration et la recherche des fonctions primitives, 2 ed., Paris, 1928.

[9] R. M. McLeod, The generalized Riemann integral, MAA, Washington, 1980.

[10] W. F. Pfeffer, The Riemann approach to integration, Cambrige Univ. Press, New York, 1993.

[11] F. Riesz and B. SZ.-Nagy, Functional analysis, 2nd. ed., Dover Publications, INC, New York, 1990, translated from the 2nd French edition by L. F. Boron.

[12] S. Saks, Theory of the integral, 2nd. rev. ed., vol. PWN, Monografie Matematyczne, Warsaw, 1937.

[13] B. S. Thomson, Real functions, Lect. Notes in Math., vol. 1170, SpringerVerlag, 1985. 
VAsile Ene 